\title{
Emittance Measurement for Beamline Extension at the PET Cyclotron
}

\author{
Sae-Hoon Park, Sang-Hoon Lee, and Yu-Seok Kim \\ Department of Energy \& Environment Engineering, Dongguk University, Gyeongju Campus, Seokjang-dong, \\ Gyeongju-si, Gyeongsangbuk-do 38066, Republic of Korea
}

Correspondence should be addressed to Yu-Seok Kim; unison@dongguk.ac.kr

Received 29 November 2015; Accepted 3 March 2016

Academic Editor: Keith E. Holbert

Copyright (C) 2016 Sae-Hoon Park et al. This is an open access article distributed under the Creative Commons Attribution License, which permits unrestricted use, distribution, and reproduction in any medium, provided the original work is properly cited.

Particle-induced X-ray emission is used for determining the elemental composition of materials. This method uses low-energy protons (of several $\mathrm{MeV}$ ), which can be obtained from high-energy (of tens $\mathrm{MeV}$ ) accelerators. Instead of manufacturing an accelerator for generating the $\mathrm{MeV}$ protons, the use of a PET cyclotron has been suggested for designing the beamline for multipurpose applications, especially for the PIXE experiment, which has a dedicated high-energy (of tens MeV) accelerator. The beam properties of the cyclotron were determined at this experimental facility by using an external beamline before transferring the ion beam to the experimental chamber. We measured the beam profile and calculated the emittance using the pepper-pot method. The beam profile was measured as the beam current using a wire scanner, and the emittance was measured as the beam distribution at the beam dump using a radiochromic film. We analyzed the measurement results and are planning to use the results obtained in the simulations of external beamline and aligned beamline components. We will consider energy degradation after computing the beamline simulation. The experimental study focused on measuring the emittance from the cyclotron, and the results of this study are presented in this paper.

\section{Introduction}

Particle-induced X-ray emission is a nondestructive technique used for determining the elemental composition of materials or samples. This method has been applied in many different fields using low-energy protons $(1-5 \mathrm{MeV})$; protons with this energy can be generated at $\mathrm{MeV}$ accelerator facilities [1]. PET cyclotrons (of $13 \mathrm{MeV}$ ) have been proposed for providing a beam of protons with energy of several $\mathrm{MeV}$ for designing the beamline for multipurpose applications. To extend their applicability, the cyclotrons can be utilized for analytical techniques that use $\mathrm{MeV}$ ion beams with energy attenuation. The PIXE beamline consists of one dipole magnet, one quadrupole doublet, and one quadrupole triplet. These magnets are used for focusing the beam to the PIXE chamber with linear dimension of about $1 \mathrm{~mm}$. The beam size should be adjusted from 1 to $10 \mathrm{~mm}$ and the beam current of $50 \mathrm{nA}$ should be obtained at the PIXE beamline target chamber.
The PIXE beamline from the cyclotron is projected to the target point in a target chamber in the experimental room. The emittance of a PET cyclotron has not been measured at the latter part of cyclotron target. The beam profile has already been measured and the beam size and the divergence were shown to be linearly related [2]. However, to extract the external beamline, we measured the beam profile and the emittance by using a diagnostic device employing a more precise measurement method. For the external beamline, the beam profile and the emittance were measured and calculated, and the results will be simulated and aligned with the beamline components. To measure properties of the cyclotron beam, we have to consider the external beamline for the PIXE experiment. This experimental paper focused on measuring the emittance of protons from the PET cyclotron. The experiment was performed at the CRC (Cyclotron Research Center) installed in Chosun University, South Korea.

Measuring the beam emittance at a PET cyclotron beamline is of fundamental importance. The traditional method 


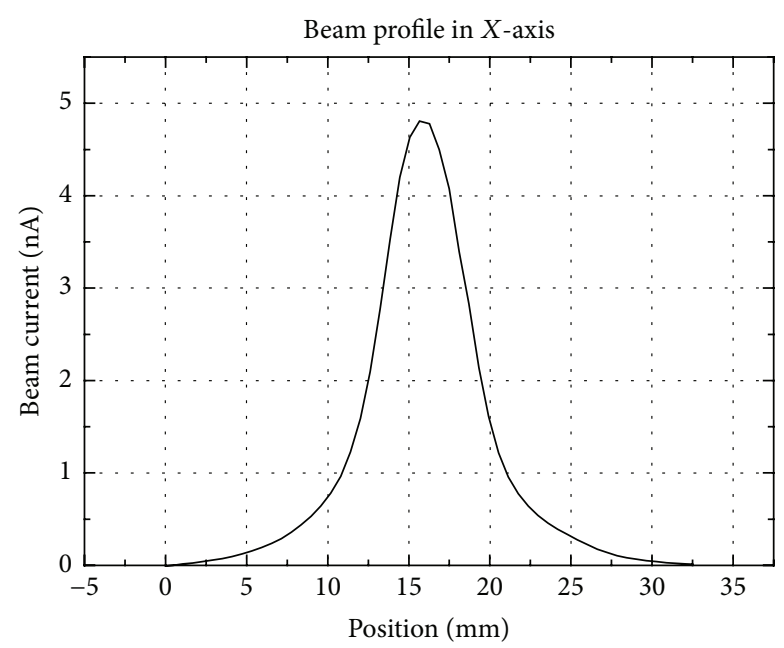

$-X$-axis

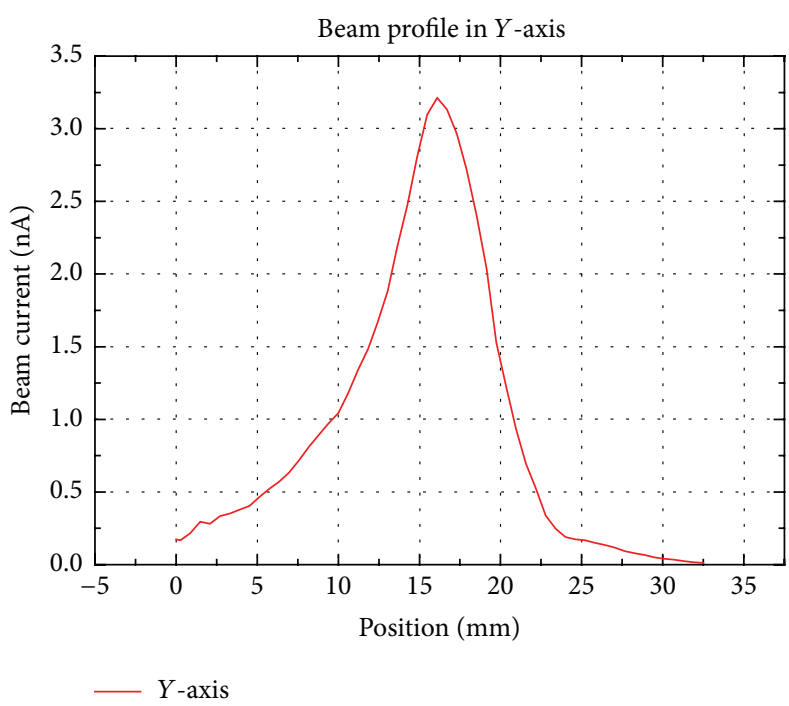

(b)

FIgURE 1: Beam profile at the cyclotron target part. (a) $x$-axis view and (b) $y$-axis view.

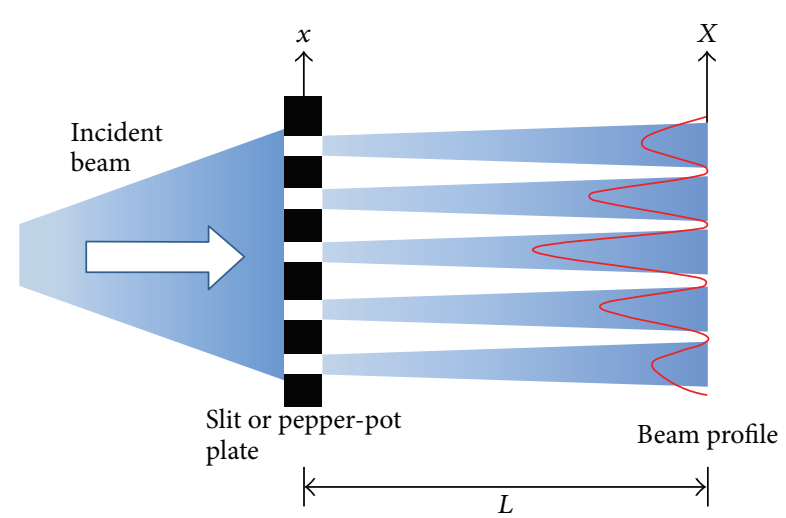

FIGURE 2: Experimental setup for emittance measurement. $L$ is the distance between the pepper-pot plate and the beam dump with the radiochromic film.

for measuring the beam profile as a function of the strength of a focusing element is not applicable, and only the slit and pepper-pot techniques can be used [3]. Two commonly used emittance measurement methods are the method of slits and the pepper-pot method. The former employs a onedimensional emittance measurement device and the latter employs a two-dimensional one [4]. In our experiments, a pepper-pot plate was installed at the cyclotron target. The wire scanner measured the beam current of the beam profile at the cyclotron target. The beam profile at the cyclotron target was measured by using the NI Labview software and equipment for controlling the motor and data acquisition for the beam current. The radiochromic film at the beam dump was also calculated by using Matlab for calculating the rms emittance.

\section{Materials and Methods}

A beam of particles can be characterized in detail by using its density in the six-dimensional phase space [5]. Beam emittance is one of the beam properties for particle accelerators. Several methods have been developed for measuring the beam emittance, such as the slit [6], pepper-pot [7, 8], and quad scan methods [9]. We used the pepper-pot method for the direct measurement of three-dimensional beam emittance. The beam profile of the initial beam at the target was acquired using the wire scanner [10], and the results are shown in Figure 1. In the case of the pepper-pot method, we analyzed the radiochromic film by using Matlab.

The method of slits employs a one-dimensional emittance measurement device, while the pepper-pot method employs a two-dimensional one [11]. The setup for the measurement based on the pepper-pot method is shown in Figure 2. The experimental setup consisted of a $2 \mathrm{~mm}$ thick pepper-pot plate comprised of carbon that was located at the cyclotron target. The thickness of the pepper-pot plate was set to $2 \mathrm{~mm}$ considering the stopping depth of $13 \mathrm{MeV}$ protons. The wire scanner was installed behind the pepper-pot plate. The wire scanner allowed measuring the beam profile of the beam that was incident on the scanner. In film measurements, the distance between the pepper-pot plate and the radiochromic film (GAFCHROMIC film, HD-810 for high density) was $209.5 \mathrm{~mm}$.

The incident beam arrived from the cyclotron and penetrated through the pepper-pot plate. The pepper-pot plate coordinate was designated as $x$, while that of the beam profile was designated by $X$. The beam size at the pepperpot plate was determined considering the dimensions of the slit appearance, the thickness of the pepper-pot plate, and the distance between the pepper-pot plate and the 


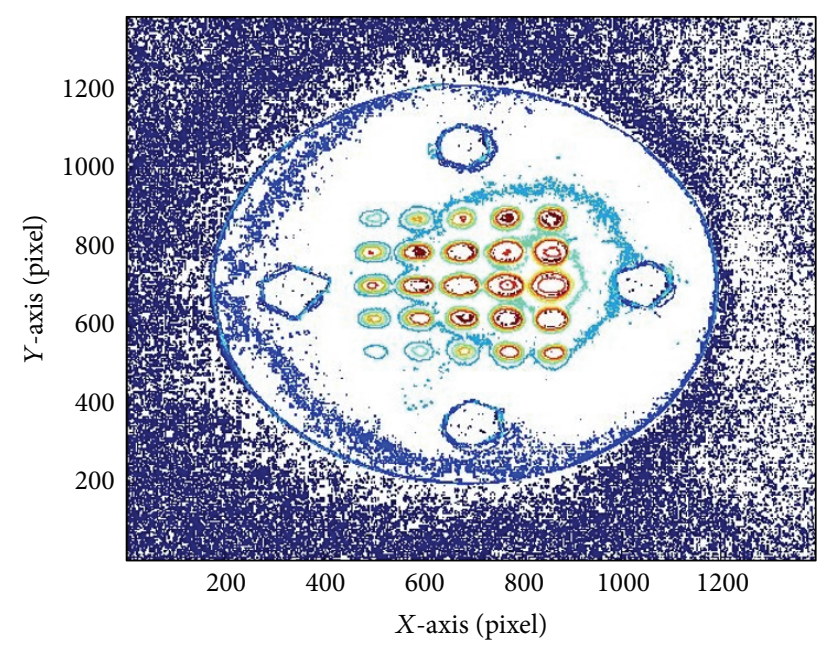

FIGURE 3: Film contour map, obtained using Matlab.

radiochromic film. The beam distribution was fitted using Gaussian functions for all profile peaks.

In the film experiment, the measurement was performed by mounting the radiochromic film on the beam dump. The hole diameters in the pepper-pot plate were $1 \mathrm{~mm}$, and the distance between adjacent holes was $2 \mathrm{~mm}$. The pepper-pot plate was $2 \mathrm{~mm}$ thick and had 25 holes. Beam irradiation time was 5 seconds and the beam current at the target was $0.13 \mu \mathrm{A}$. Longer irradiation times could result in film damage, thereby destroying the image. Film measurement was performed with short irradiation time for reducing the film image error. The film analysis was performed using Matlab.

\section{Results and Discussion}

The film intensity data were encoded as double precision numbers. In turn, these data were converted to the RGB format, with brightness ranging from 0 to 1 . The film image resolution was 600 dots per inch (DPI) and the image size was $1392 \times 1392$ pixels. The size of one pixel was $42.33 \mu \mathrm{m}$. The contour map was created using the film data in the RGB format and is shown in Figure 3.

The film data were divided into rows $(x)$ and columns $(y)$. The sum of all values in a certain row represents the emittance along the $y$ axis, and the sum of all values in a certain column represents the emittance along the $x$ axis. All rows and columns exhibited five peaks that were fitted with Gaussian functions in Matlab. Three parameters-the intensity, peak position, and peak sigma-were computed from these Gaussian fits of the film data. The emittance was calculated as [12]

$$
\epsilon=\sqrt{\left\langle x^{2}\right\rangle\left\langle x^{\prime 2}\right\rangle-\left\langle x x^{\prime}\right\rangle^{2}}
$$

The rms emittance was calculated from the Gaussian fits in Matlab, for both $x$ - and $y$-directions. The rms emittance values were $40.401 \pi \mathrm{mm}$ mrad for the $x$-direction and $47.431 \pi \mathrm{mm}$ mrad for the $y$-direction. We also computed the beam twiss parameters from the calculated values of rms

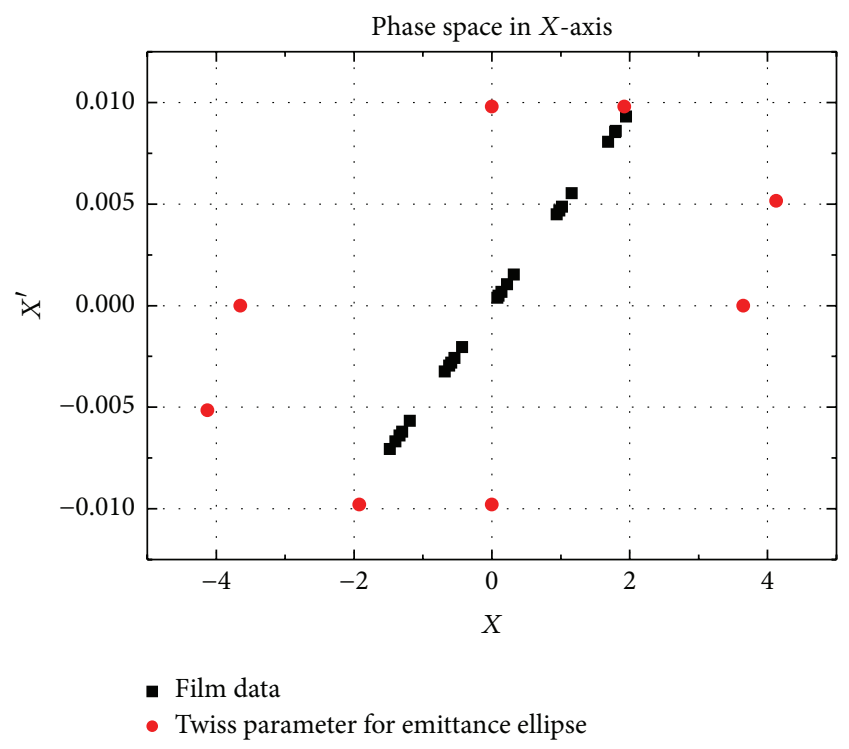

(a)

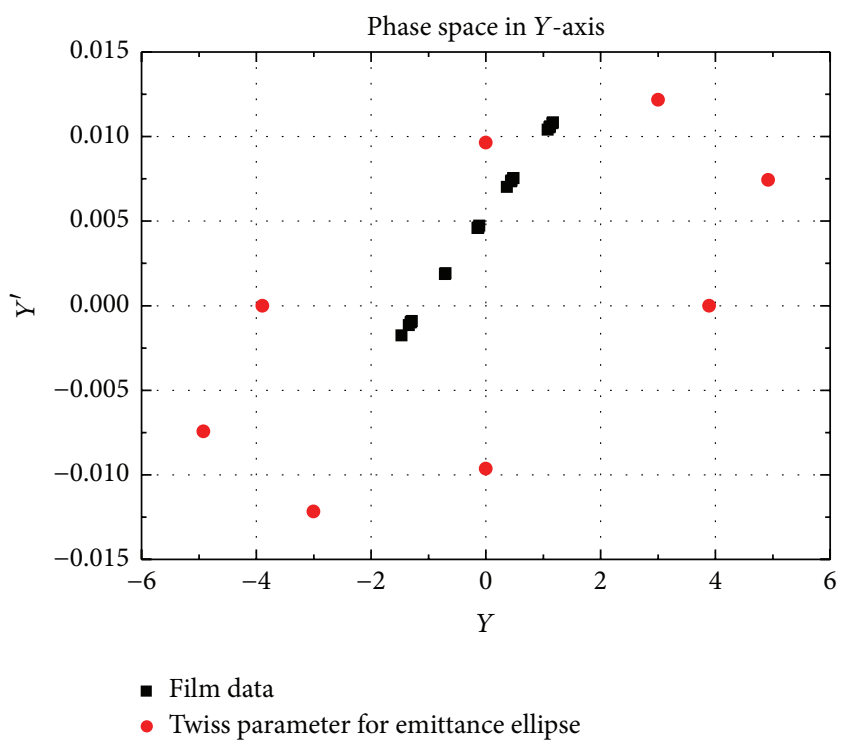

(b)

Figure 4: Phase space ellipse. (a) $X$-coordinate and (b) $Y$ coordinate.

emittance. These values determine the shape and orientation of the beam ellipse in the phase space [13]. The phase space ellipses are shown in Figure 4. The red dots delimit the maximal area in the phase space [14], while the black dots enclosed in the red dots are the film data. These results will be used to estimate the beamline simulations.

\section{Conclusions}

The CRC has been proposed to be used for producing an external beamline for experimental research. To use the existing facility for producing PET cyclotron protons as an external beamline, the beam energy should be reduced to several $\mathrm{MeV}$. There is no correlation between beam emittance 
and energy degradation at the end of beamline. To generate an external beamline, experiments were conducted using the specific data of beam emittance at the latter part of cyclotron target by a more precise measurement method. The beam size was measured with the wire scanner. The rms emittance was calculated from the Gaussian fits parameters and phase space ellipse. The beam emittance was measured for providing conditioning parameters for design and simulations of beamline components of the external beamline for obtaining the desired beam shape.

\section{Competing Interests}

The authors declare that they have no competing interests.

\section{Acknowledgments}

This research was supported by the National Nuclear R\&D Program through the National Research Foundation of Korea (NRF) funded by the Ministry of Science, ICT and Future Planning (2015M2B2A6030404). The authors would like to express their deepest gratitude to the CRC of Chosun University for their help in conducting the experiment.

\section{References}

[1] K. Ishii and S. Morita, "Theoretical estimation of PIXE detection limits," Nuclear Instruments and Methods in Physics Research Section B: Beam Interactions with Materials and Atoms, vol. 34, no. 2, pp. 209-216, 1988.

[2] S.-H. Park and Y.-S. Kim, "Characterization of the beam profile for the PIXE/PIGE beam line at the CRC proton cyclotron," Journal of the Korean Physical Society, vol. 63, no. 7, pp. 12911295, 2013.

[3] C. H. Wang, M. Zhang, M. Clausen, and Z. Kakucs, "Slits measurement of emittance on TTF" in Proceedings of the 7th International Conference on Accelerator and Large Experimental Physics Control Systems, Trieste, Italy, October 1999.

[4] M. Zhang, "Emittance formula for slits and pepper-pot measurement," Tech. Rep. FERMILAB-TM-1998, Fermi National Accelerator Laboratory, Batavia, Ill, USA, 1998.

[5] K. T. McDonald and D. P. Russell, Methods of Emittance Measurement, Joseph Henry Laboratories, Princeton University, Princeton, NJ, USA, 1988.

[6] C. Liu, D. Gassner, M. Minty, and P. Thieberger, Multi-Slit Based Emittance Measurement Study for BNL ERL, ColliderAccelerator Department Brookhaven National Laboratory, Upton, NY, USA, 2012.

[7] H. Takeda and J. H. Billen, "PARMILA," Los Alamos National Laboratory Report LA-UR-98-4478, Los Alamos National Laboratory, Los Alamos, NM, USA, 2004.

[8] S. Y. Lee, Accelerator Physics, World Scientific Publishing Company, New York, NY, USA, 2nd edition, 2004.

[9] J. G. Wang, D. X. Wang, and M. Reiser, "Beam emittance measurement by the pepper-pot method," Nuclear Instruments and Methods in Physics Research A, vol. 307, no. 2-3, pp. 190-194, 1991.

[10] P. N. Ostroumov, P. Billquist, M. Portillo, and W. Q. Shen, "Design and test of a beam profile monitoring device for low intensity radioactive beams," Review of Scientific Instruments, vol. 73, no. 1, p. 56, 2002.

[11] R. Hollinger, K. Volk, and H. Klein, "Measurement of the beam emittance of the Frankfurt proton source," Review of Scientific Instruments, vol. 73, no. 2, p. 1027, 2002.

[12] T. P. Wangler, RF Linear Accelerators, John Wiley \& Sons, New York, NY, USA, 2nd edition, 1998.

[13] N. Tongnopparat, L. D. Yu, T. Vilaithong, and H. Wiedemann, "Ion beam emittance measurement based on transformation matrix theory," Journal of the Korean Physical Society, vol. 53, no. 6, pp. 3744-3748, 2008.

[14] S. Kondrashev, A. Barcikowski, B. Mustapha, P. N. Ostroumov, and N. Vinogradov, "Development of a pepper pot emittance probe and its application for ECR ion beam studies," Nuclear Instruments and Methods in Physics Research Section A: Accelerators, Spectrometers, Detectors and Associated Equipment, vol. 606, no. 3, pp. 296-304, 2009. 

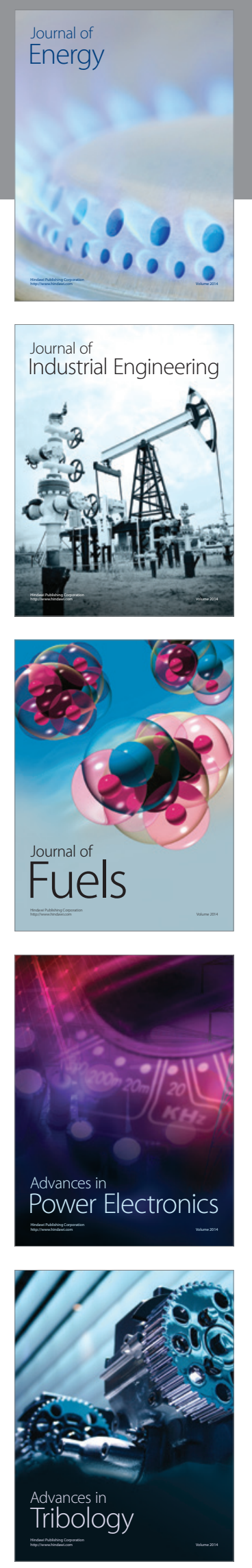
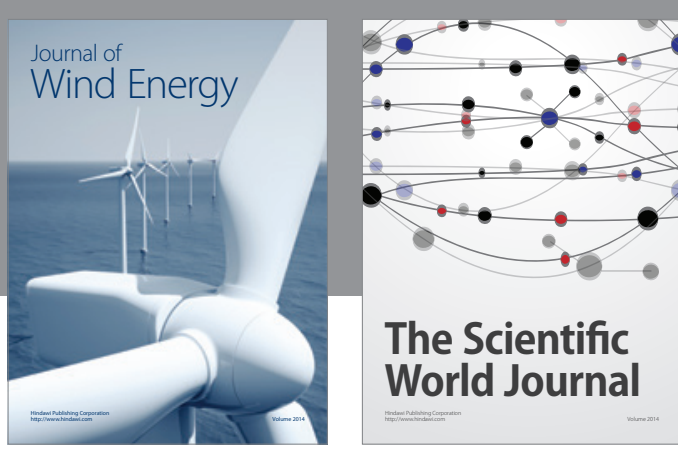

The Scientific World Journal
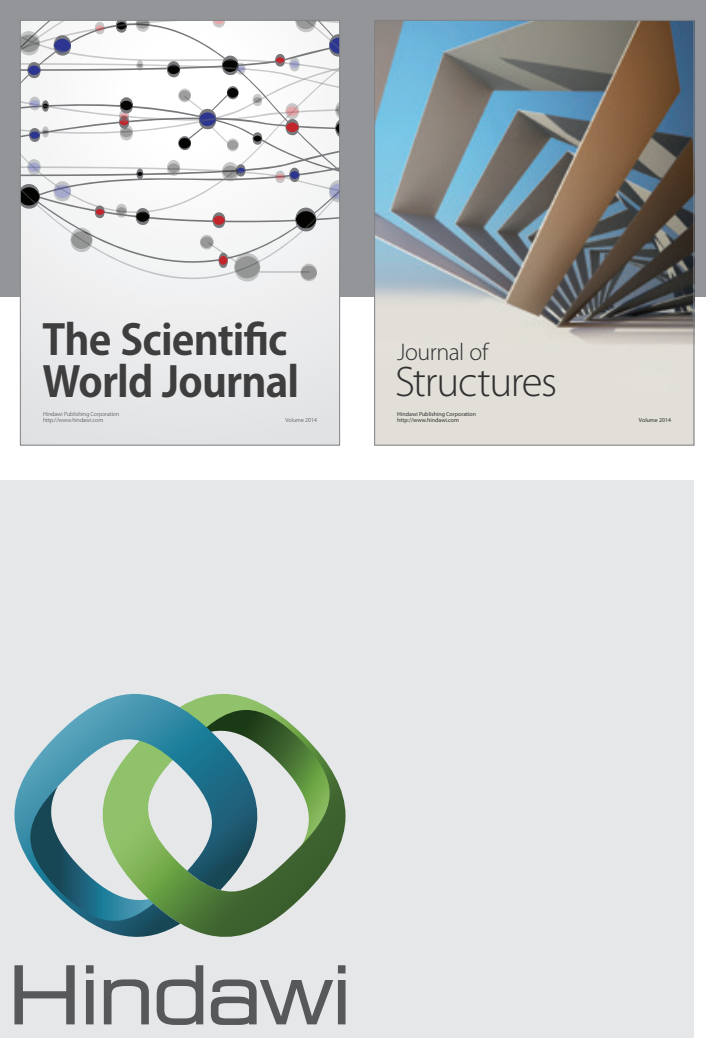

Submit your manuscripts at

http://www.hindawi.com
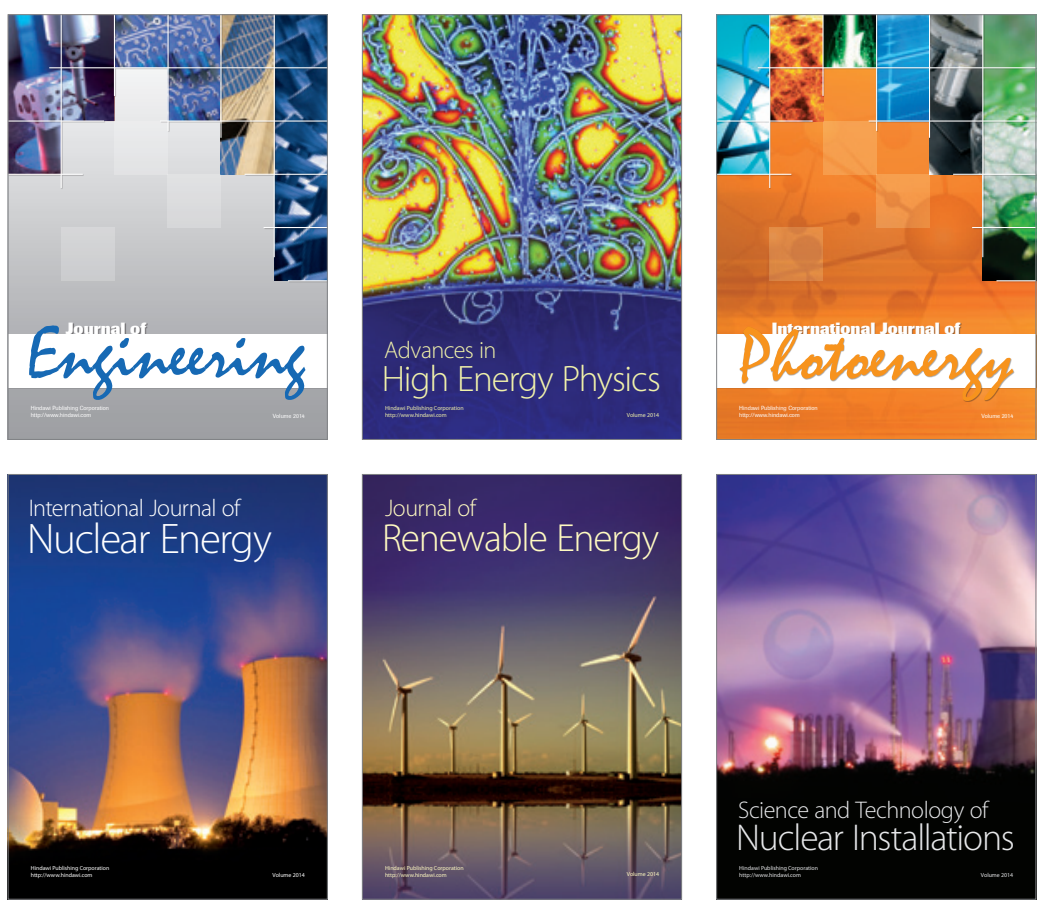
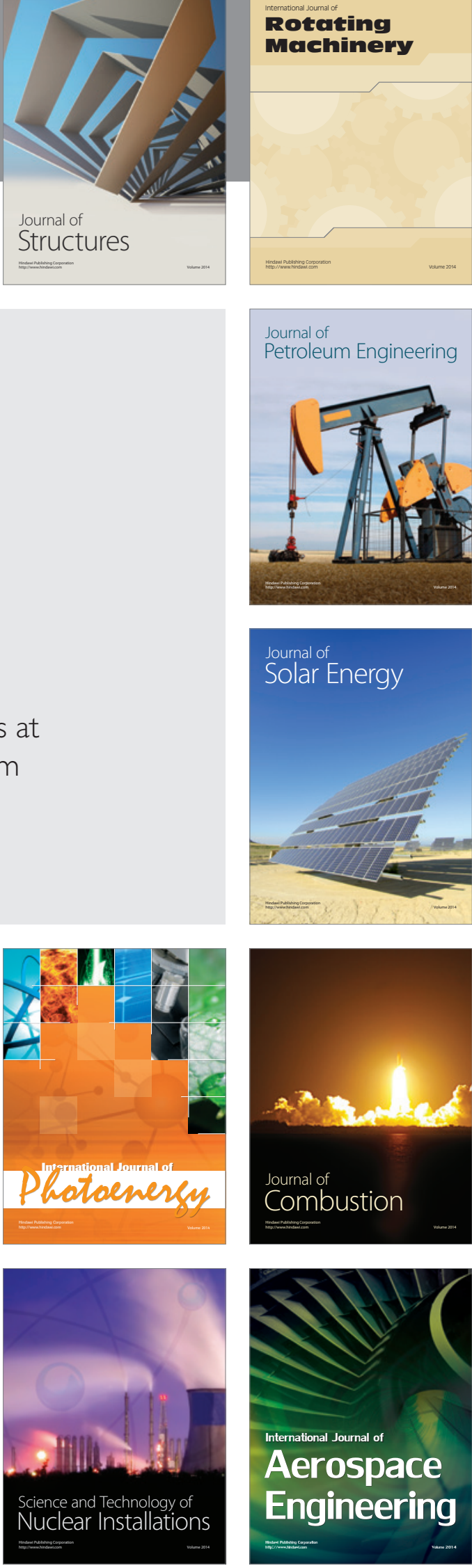